\title{
COMPLEX SURFACES WITH EQUIVALENT DERIVED CATEGORIES
}

\author{
TOM BRIDGELAND AND ANTONY MACIOCIA
}

\begin{abstract}
We examine the extent to which a smooth minimal complex projective surface $X$ is determined by its derived category of coherent sheaves $\mathrm{D}(X)$. To do this we find, for each such surface $X$, the set of surfaces $Y$ for which there exists a Fourier-Mukai transform $\mathrm{D}(Y) \rightarrow \mathrm{D}(X)$.
\end{abstract}

\section{INTRODUCTION}

This paper addresses the question: to what extent is a smooth projective variety $X$ determined by its bounded derived category of coherent sheaves $\mathrm{D}(X)$ ? Recall [14] that $\mathrm{D}(X)$ is a triangulated category, whose objects are bounded complexes of coherent sheaves on $X$. If $Y$ is another smooth projective variety, an equivalence of categories

$$
\Phi: \mathrm{D}(Y) \longrightarrow \mathrm{D}(X)
$$

preserving the triangles is called a Fourier-Mukai transform. Put another way then, our problem is to find, for a given variety $X$, the set of Fourier-Mukai partners of $X$, i.e. the set of varieties $Y$ for which there exists a Fourier-Mukai transform relating $X$ and $Y$.

This problem is interesting for several reasons. Firstly, FourierMukai (FM) transforms have shown themselves to be important tools for studying moduli spaces of sheaves [7], 17], [21], and it is therefore natural to attempt to classify them. Secondly, the theory of FourierMukai-type transforms seems to provide the correct language for describing certain geometrical dualities suggested by string theory. As a particular example of this, M. Kontsevich's homological mirror conjecture [16] predicts that all mirror varieties of a given variety have equivalent derived categories. Thus, the existence of distinct FM partners of a variety $X$ may relate to the possibility that the conjectural mirror map is not a well-defined bijection at $X$.

The first example of a non-trivial FM transform was given by $\mathrm{S}$. Mukai in 1981 and related the derived category of an abelian variety with the derived category of the dual variety [19]. Since then further examples have been given, involving K3 surfaces [3], 8], abelian surfaces [17], elliptic surfaces [7] and Enriques and bielliptic surfaces [10]. Clearly, some sort of classification is in order. 
The classification of FM transforms splits naturally into two parts. Given a smooth projective variety $X$ these are

(a) find the set of FM partners of $X$, that is, the set of varieties $Y$ for which there exists a FM transform $\mathrm{D}(Y) \rightarrow \mathrm{D}(X)$,

(b) find the group of FM transforms $\mathrm{D}(X) \rightarrow \mathrm{D}(X)$.

When $X$ has ample canonical or anticanonical bundle a complete solution was obtained by A. Bondal and D. Orlov [5], [6]. In this case the answer is rather trivial, in that the only FM partner of $X$ is $X$ itself, and all autoequivalences of $\mathrm{D}(X)$ are generated by shifts, automorphisms of $X$ and twists by line bundles.

Remarkably, Orlov also managed to solve both problems when $X$ is an abelian variety. In this case the solution is very interesting and highly non-trivial [25].

These two results together give a simple answer to both problems in the case when $X$ has dimension one. In particular, it is possible to prove that the only FM partner of a curve $X$ is $X$ itself.

In this paper we solve Problem (a) for minimal complex surfaces. We obtain the following theorem, which will be explained in greater detail below.

Theorem 1.1. Let $X$ be a smooth minimal complex projective surface, and let $Y$ be a Fourier-Mukai partner of $X$, not isomorphic to $X$. Then either $X$ is an elliptic surface, and $Y$ is another elliptic surface obtained as in [7], [13], by taking a relative Picard scheme of the elliptic fibration on $X$, or $X$ is of $K 3$ or abelian type, and $Y$ is of the same type, with Hodge-isometric transcendental lattice.

Corollary 1.2. The number of FM partners of a smooth minimal complex projective surface is finite.

The proof of Theorem 1.1 is rather long, since each different type of surface appearing in the Enriques classification must be analysed separately. For surfaces of Kodaira dimension 0, the problem is mostly lattice-theoretic, and we rely heavily on results of V. Nikulin. Other surfaces are best treated with more geometric methods. In particular, it becomes important to classify curves with non-positive self-intersection which do not intersect the canonical divisor.

Problem (b) for surfaces is much more difficult. In particular, determining the group of autoequivalences of the derived category of a K3 surface seems to be of considerable interest.

Notation. All varieties will be over $\mathbb{C}$. Given a variety $X$, the translation (or shift) functor on $\mathrm{D}(X)$ is written [1], so that the symbol $E[m]$ means the object $E$ of $\mathrm{D}(X)$ shifted to the left by $m$ places. By a sheaf on $X$ we mean a coherent $\mathcal{O}_{X}$-module, and a point of $X$ always means a closed (or geometric) point. The structure sheaf of such a point $x \in X$ 
will be denoted $\mathcal{O}_{x}$. The canonical bundle of a smooth projective variety $X$ is denoted $\omega_{X}$. By a lattice we mean a free abelian group of finite rank with a non-degenerate $\mathbb{Z}$-valued symmetric bilinear form.

\section{Preliminaries on Fourier-Mukai transforms}

Throughout this section we fix a pair of smooth projective varieties $X$ and $Y$.

2.1. A Fourier-Mukai transform relating $X$ and $Y$ is an exact 1 equivalence of categories

$$
\Phi: \mathrm{D}(Y) \longrightarrow \mathrm{D}(X) .
$$

Due to a theorem of Orlov [24], it is known that for any such equivalence $\Phi$ there is an object $\mathcal{P}$ of $\mathrm{D}(Y \times X)$, unique up to isomorphism, such that $\Phi$ is isomorphic to the functor defined by the formula

$$
\Phi_{Y \rightarrow X}^{\mathcal{P}}(-)=\mathbf{R} \pi_{X, *}\left(\mathcal{P} \stackrel{\mathbf{L}}{\otimes} \pi_{Y}^{*}(-)\right),
$$

where $Y \stackrel{\pi_{Y}}{\longleftarrow} Y \times X \stackrel{\pi_{X}}{\longrightarrow} X$ are the projection maps. The object $\mathcal{P}$ is called the kernel of the transform $\Phi$.

We say that $X$ and $Y$ are Fourier-Mukai partners if there is a FM transform relating $X$ and $Y$. This is equivalent to the statement that $\mathrm{D}(X)$ and $\mathrm{D}(Y)$ are equivalent as triangulated categories.

Lemma 2.1. If $X$ and $Y$ are $F M$ partners then $\operatorname{dim}(X)=\operatorname{dim}(Y)$ and the canonical line bundles $\omega_{X}$ and $\omega_{Y}$ have the same order.

Proof. Define the Serre functor $\mathrm{S}_{X}$ on the category $\mathrm{D}(X)$ by the formula

$$
\mathrm{S}_{X}(-)=\omega_{X} \otimes(-)[\operatorname{dim} X] .
$$

In [4, Prop. 3.4] it is shown that any FM transform

$$
\Phi: \mathrm{D}(Y) \longrightarrow \mathrm{D}(X)
$$

commutes with the Serre functors on $X$ and $Y$. Thus if $\Psi$ is a quasiinverse to the equivalence $\Phi$, there is an isomorphism of functors

$$
\mathrm{S}_{Y} \cong \Psi \circ \mathrm{S}_{X} \circ \Phi \text {. }
$$

The lemma is an immediate consequence of this.

Given a FM transform $\Phi: \mathrm{D}(Y) \rightarrow \mathrm{D}(X)$, and an object $E$ of $\mathrm{D}(Y)$, let us write

$$
\Phi^{i}(E)=\mathrm{H}^{i}(\Phi(E))
$$

for the $i$ th cohomology sheaf of the object $\Phi(E)$ of $\mathrm{D}(X)$. We shall call $\Phi$ a sheaf transform if there is an integer $p$ such that for each point $y \in Y$,

$$
\Phi^{i}\left(\mathcal{O}_{y}\right)=0 \text { unless } i=p .
$$

\footnotetext{
${ }^{1} \mathrm{~A}$ functor between triangulated categories is exact if it commutes with the translation functors, and takes distinguished triangles to distinguished triangles.
} 
An equivalent condition, [8, Lemma 4.3], is that the kernel of $\Phi$ is concentrated in some degree $p$, and is flat over $Y$.

2.2. Let $E$ and $F$ be objects of $\mathrm{D}(Y)$. For each integer $i$ one defines a vector space

$$
\operatorname{Hom}_{\mathrm{D}(Y)}^{i}(E, F)=\operatorname{Hom}_{\mathrm{D}(Y)}(E, F[i]) .
$$

Recall that if $E$ and $F$ are concentrated in degree 0 then these spaces are just the Ext-groups of the sheaves $E$ and $F$, i.e.

$$
\operatorname{Hom}_{\mathrm{D}(Y)}^{i}(E, F)=\operatorname{Ext}_{Y}^{i}(E, F) .
$$

The following trivial but useful observation is sometimes referred to as the Parseval theorem.

Lemma 2.2. Let $\Phi: \mathrm{D}(Y) \rightarrow \mathrm{D}(X)$ be a FM transform, and take objects $E$ and $F$ of $\mathrm{D}(Y)$. Then

$$
\operatorname{Hom}_{\mathrm{D}(X)}^{i}(\Phi(E), \Phi(F))=\operatorname{Hom}_{\mathrm{D}(Y)}^{i}(E, F) .
$$

Proof. Immediate because $\Phi$ is an equivalence of categories, commuting with the translation functors in $\mathrm{D}(Y)$ and $\mathrm{D}(X)$.

The lemma implies that

$$
\chi(\Phi(E), \Phi(F))=\chi(E, F),
$$

where $\chi(E, F)$ denotes the relative Euler character

$$
\chi(E, F)=\sum_{i}(-1)^{i} \operatorname{dim} \operatorname{Hom}_{\mathrm{D}(Y)}^{i}(E, F) .
$$

This relative Euler character is given in terms of the Chern characters2 of $E$ and $F$ by the Riemann-Roch theorem. For example, if $Y$ is a surface, then

$$
\begin{aligned}
\chi(E, F)= & \mathrm{r}(E) \operatorname{ch}_{2}(F)-\mathrm{c}_{1}(E) \cdot \mathrm{c}_{1}(F)+\mathrm{r}(F) \mathrm{ch}_{2}(E) \\
& +\frac{1}{2}\left(\mathrm{r}(F) \mathrm{c}_{1}(E)-\mathrm{r}(E) \mathrm{c}_{1}(F)\right) \cdot K_{Y}+\mathrm{r}(E) \mathrm{r}(F) \chi\left(\mathcal{O}_{Y}\right),
\end{aligned}
$$

where $K_{Y}$ is the first Chern class of the canonical line bundle $\omega_{Y}$. In particular, if $E$ and $F$ are torsion sheaves

$$
\chi(E, F)=-\mathrm{c}_{1}(E) \cdot \mathrm{c}_{1}(F) .
$$

\footnotetext{
${ }^{2}$ The Chern character of an object of the derived category is just the alternating sum of the Chern characters of the cohomology sheaves.
} 
2.3. Grothendieck's Riemann-Roch theorem implies that for any FM transform $\Phi: \mathrm{D}(Y) \rightarrow \mathrm{D}(X)$ there is a linear map of vector spaces

$$
\phi: \mathrm{H}^{*}(Y, \mathbb{Q}) \longrightarrow \mathrm{H}^{*}(X, \mathbb{Q})
$$

making the following diagram commute

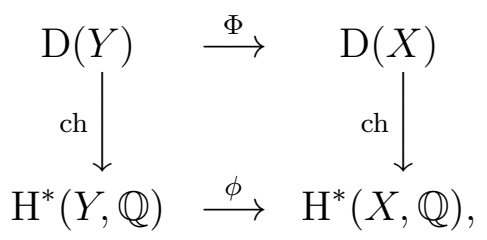

where ch denotes the operation of taking the Chern character.

The proof of [22, Theorem 4.9] shows that $\phi$ is an isomorphism of vector spaces. Furthermore, since $\phi$ is given by an algebraic class on the product $Y \times X$, it preserves the parity of the degree of cohomology classes. One therefore has

Proposition 2.3. Surfaces with equivalent derived categories have the same Picard number, and the same topological Euler number.

2.4. An important property of FM transforms is that they preserve families of sheaves. Let $\Phi: \mathrm{D}(Y) \rightarrow \mathrm{D}(X)$ be a FM transform, take a scheme $S$ of finite type over $\mathbb{C}$, and let $\mathcal{E}$ be a sheaf on $S \times Y$, flat over $S$.

Proposition 2.4. The set of points $s \in S$ for which the object $\Phi\left(\mathcal{E}_{s}\right)$ of $\mathrm{D}(X)$ is concentrated in degree 0 is the set of points of an open subset $U$ of $S$ (possibly empty). Furthermore there is a sheaf $\mathcal{F}$ on $U \times X$, flat over $U$, such that for any point $s \in U, \mathcal{F}_{s}=\Phi\left(\mathcal{E}_{s}\right)$.

Proof. See [9, Chapter 6] or [21, Theorem 1.6].

As a consequence one has

Lemma 2.5. Let $\Phi: \mathrm{D}(Y) \rightarrow \mathrm{D}(X)$ be a FM transform, and suppose there is a point $y \in Y$, such that

$$
\Phi\left(\mathcal{O}_{y}\right)=\mathcal{O}_{x}[p],
$$

for some point $x \in X$ and some integer $p$. Then $X$ and $Y$ are birationally equivalent.

Proof. By Prop. 2.4 there is an open subset $V \subset Y$ such that for each point $y \in V$, there is a point $f(y) \in X$ with

$$
\Phi\left(\mathcal{O}_{y}\right)=\mathcal{O}_{f(y)}[p] .
$$

The kernel of $\Phi$, restricted to $V \times X$, is supported on the graph of $f$, so $f$ is a morphism $V \rightarrow X$, and hence defines a birational map $Y \rightarrow X$. Since $\Phi$ is an equivalence this birational map has an inverse, so $X$ and $Y$ are birationally equivalent. 
Remark 2.6. Suppose the conditions of Lemma 2.5 hold, and that $X$ is a minimal surface of non-negative Kodaira dimension. Then $Y$ must be the blow-up of $X$ at $r \geq 0$ points. But by Prop. 2.3, $X$ and $Y$ have the same Picard number, so $r=0$, and $X$ and $Y$ are isomorphic.

2.5. The following important result allows one to construct examples of FM transforms. It was proved by Bondal and Orlov [5], and one of us [8], using ideas of Mukai.

Theorem 2.7. Suppose $X$ and $Y$ have dimension $n$. Let $\mathcal{P}$ be an object of $\mathrm{D}(Y \times X)$, and let $\Phi$ denote the exact functor

$$
\Phi_{Y \rightarrow X}^{\mathcal{P}}: \mathrm{D}(Y) \longrightarrow \mathrm{D}(X)
$$

defined above. Then $\Phi$ is an equivalence of categories if and only if, for each point $y \in Y$,

$$
\operatorname{Hom}_{\mathrm{D}(X)}\left(\Phi\left(\mathcal{O}_{y}\right), \Phi\left(\mathcal{O}_{y}\right)\right)=\mathbb{C} \text { and } \Phi\left(\mathcal{O}_{y}\right) \otimes \omega_{X} \cong \Phi \mathcal{O}_{y},
$$

and for each pair of points $y_{1}, y_{2} \in Y$, and each integer $i$,

$$
\operatorname{Hom}_{\mathrm{D}(X)}^{i}\left(\Phi\left(\mathcal{O}_{y_{1}}\right), \Phi\left(\mathcal{O}_{y_{2}}\right)\right)=0 \text { unless } y_{1}=y_{2} \text { and } 0 \leq i \leq n \text {. }
$$

Most examples of FM transforms for surfaces arise via the following simple corollary. Recall that a sheaf on a smooth projective variety $X$ is called special if $E \otimes \omega_{X}=E$.

Corollary 2.8. Let $X$ be a smooth projective surface with a fixed polarisation, and let $Y$ be a smooth, fine, complete, two-dimensional moduli space of special, stable sheaves on $X$. Then there is a universal sheaf $\mathcal{P}$ on $Y \times X$, and the functor $\Phi_{Y \rightarrow X}^{\mathcal{P}}$ is a FM transform.

Proof. The assumption that $Y$ is fine means that there is a universal sheaf $\mathcal{P}$ on $Y \times X$, flat over $Y$. For each point $y \in Y, \mathcal{P}_{y}$ is a stable (hence simple), special sheaf on $X$. Furthermore, since $Y$ is smooth of dimension 2, the tangent space to $Y$ at $y$, which is given by

$$
\operatorname{Ext}_{X}^{1}\left(\mathcal{P}_{y}, \mathcal{P}_{y}\right)
$$

has dimension 2. It follows that for any pair of points $y_{1}, y_{2} \in Y$,

$$
\chi\left(\mathcal{P}_{y_{1}}, \mathcal{P}_{y_{2}}\right)=1-2+1=0 .
$$

If $y_{1}$ and $y_{2}$ are distinct then there are no non-zero maps between the sheaves $\mathcal{P}_{y_{1}}$ and $\mathcal{P}_{y_{2}}$, so by Serre duality,

$$
\operatorname{Ext}_{X}^{2}\left(\mathcal{P}_{y_{1}}, \mathcal{P}_{y_{2}}\right)=\operatorname{Hom}_{X}\left(\mathcal{P}_{y_{2}}, \mathcal{P}_{y_{1}}\right)^{\vee}=0
$$

The result then follows from Theorem 2.7. 
2.6. Assume that $X$ and $Y$ are surfaces. Our basic tool for classifying FM transforms is

Lemma 2.9. Let $\Phi: \mathrm{D}(Y) \rightarrow \mathrm{D}(X)$ be a FM transform and take a point $y \in Y$. Then there is an inequality

$$
\sum_{i} \operatorname{dim} \operatorname{Ext}_{X}^{1}\left(\Phi^{i}\left(\mathcal{O}_{y}\right), \Phi^{i}\left(\mathcal{O}_{y}\right)\right) \leq 2
$$

and moreover, each of the sheaves $\Phi^{i}\left(\mathcal{O}_{y}\right)$ is special.

Proof. The second statement is immediate from Theorem 2.7. For the first part consider the spectral sequence [5, Prop. 4.2],

$$
E_{2}^{p, q}=\bigoplus_{i} \operatorname{Ext}_{X}^{p}\left(\Phi^{i}\left(\mathcal{O}_{y}\right), \Phi^{i+q}\left(\mathcal{O}_{y}\right)\right) \Longrightarrow \operatorname{Hom}_{\mathrm{D}(X)}^{p+q}\left(\Phi\left(\mathcal{O}_{y}\right), \Phi\left(\mathcal{O}_{y}\right)\right) .
$$

The $E_{2}^{1,0}$ term survives to infinity, and by Lemma 2.2

$$
\operatorname{Hom}_{\mathrm{D}(X)}^{1}\left(\Phi\left(\mathcal{O}_{y}\right), \Phi\left(\mathcal{O}_{y}\right)\right)=\operatorname{Hom}_{\mathrm{D}(Y)}^{1}\left(\mathcal{O}_{y}, \mathcal{O}_{y}\right)=\mathbb{C}^{2},
$$

so the result follows.

Corollary 2.10. Suppose $X$ is an abelian surface. Then every FM transform $\Phi: \mathrm{D}(Y) \rightarrow \mathrm{D}(X)$ is a sheaf transform.

Proof. For any non-zero sheaf $E$ on an abelian surface $X$, the dimension of the space $\operatorname{Ext}_{X}^{1}(E, E)$ is at least 2 .

2.7. The support of an object $E$ of $\mathrm{D}(X)$ is defined to be the union of the supports of the cohomology sheaves of $E$. It is a closed subset of $X$. A point $x \in X$ lies in the support of an object $E$ of $\mathrm{D}(X)$ if and only if there is an integer $i$ such that

$$
\operatorname{Hom}_{\mathrm{D}(X)}^{i}\left(E, \mathcal{O}_{x}\right) \neq 0 .
$$

This statement follows from a simple spectral sequence argument [8, Ex. 2.2].

Suppose $\Phi: \mathrm{D}(Y) \rightarrow \mathrm{D}(X)$ is a FM transform, and let $\Psi$ be a quasiinverse $\mathrm{D}(X) \rightarrow \mathrm{D}(Y)$. Let $n$ be the common dimension of $X$ and $Y$. For any pair of points $(y, x) \in Y \times X$,

$$
\begin{aligned}
\operatorname{Hom}_{\mathrm{D}(Y)}^{i}\left(\Psi\left(\mathcal{O}_{x}\right), \mathcal{O}_{y}\right) & =\operatorname{Hom}_{\mathrm{D}(X)}^{i}\left(\mathcal{O}_{x}, \Phi\left(\mathcal{O}_{y}\right)\right) \\
& =\operatorname{Hom}_{\mathrm{D}(X)}^{n-i}\left(\Phi\left(\mathcal{O}_{y}\right), \mathcal{O}_{x}\right)^{\vee},
\end{aligned}
$$

so $x$ lies in the support of $\Phi\left(\mathcal{O}_{y}\right)$ precisely when $y$ lies in the support of $\Psi\left(\mathcal{O}_{x}\right)$.

A simple consequence of this is that the supports of the objects $\Phi\left(\mathcal{O}_{y}\right)$, as $y$ varies in $Y$, cover $X$. For otherwise there would be a point $x \in X$ such that $\Psi\left(\mathcal{O}_{x}\right)$ had empty support, and hence was zero, contradicting the assumption that $\Psi$ is an equivalence. An extension of this argument gives 
Lemma 2.11. Let $X$ and $Y$ be surfaces, and $\Phi: \mathrm{D}(Y) \rightarrow \mathrm{D}(X)$ a $F M$ transform. Suppose $X$ has non-zero Kodaira dimension, and take a finite set of points $S \subset X$. Then for a general point $y \in Y$, the support of $\Phi\left(\mathcal{O}_{y}\right)$ is disjoint from $S$.

Proof. Assume the opposite. Then every point of $Y$ lies in the union over $x \in S$ of the supports of the objects $\Psi\left(\mathcal{O}_{x}\right)$, so for some $x \in S$, the support of $\Psi\left(\mathcal{O}_{x}\right)$ is the whole of $Y$. Since each cohomology sheaf of $\Psi\left(\mathcal{O}_{x}\right)$ is special, it follows that $\omega_{Y}$ has finite order, contradicting Lemma 2.1.

\section{Ruled SuRfaCes AND SURFACES OF GENERAL TYPE}

We start our classification of Fourier-Mukai transforms by looking at surfaces with Kodaira dimension $-\infty$ and 2 .

Proposition 3.1. Let $X$ be a minimal surface of general type. Then the only FM partner of $X$ is $X$ itself.

Proof. It is a standard fact [2, VII.2.3, VII.2.5] that $X$ has only finitely many irreducible curves $D$ satisfying $D \cdot K_{X}=0$. Thus, by Lemma 2.11, given a FM transform $\Phi: \mathrm{D}(Y) \rightarrow \mathrm{D}(X)$, we may choose $y \in Y$ so that the support of $\Phi\left(\mathcal{O}_{y}\right)$ does not contain any of these curves.

Let $E$ be a non-zero cohomology sheaf of $\Phi\left(\mathcal{O}_{y}\right)$. Since $E$ is special, $E$ is a torsion sheaf, and $\mathrm{c}_{1}(E) \cdot K_{X}=0$, so $c_{1}(E)=0$, and $E$ is supported in dimension zero. Then Riemann-Roch gives $\chi(E, E)=0$, and this implies that $\operatorname{Ext}_{X}^{1}(E, E)$ has dimension at least 2. This applies to any cohomology sheaf of $\Phi\left(\mathcal{O}_{y}\right)$ so Lemma 2.9 implies that some shift of $\Phi\left(\mathcal{O}_{y}\right)$ is a sheaf $E$. Then, by Lemma 2.2, $E$ is simple, hence isomorphic to $\mathcal{O}_{x}$ for some $x \in X$, and Remark 2.6 shows that $Y$ is isomorphic to $X$.

Proposition 3.2. Let $X$ be a minimal surface of Kodaira dimension $-\infty$ with no elliptic fibration. Then the only FM partner of $X$ is $X$ itself.

Proof. Let $\Phi: \mathrm{D}(Y) \rightarrow \mathrm{D}(X)$ be a FM transform. If $X=\mathbb{P}^{2}$ then the argument of Prop. 3.1 applies, so we may take $X$ to be a ruled surface over a curve of genus $g$.

We freely use notation and results from [15, §V.2]. Recall in particular that the Néron-Severi group of $X$ is generated by two elements $C$ and $f$, satisfying

$$
C^{2}=-e, \quad f^{2}=0, \quad C \cdot f=1,
$$

where $e$ is some integer invariant of $X$. In terms of this basis

$$
K_{X}=-2 C+(2 g-2-e) f .
$$

We shall assume for the moment that $X$ is not the unique rational ruled surface with invariant $e=2$. 
Suppose $D$ is an irreducible curve on $X$ with $D \cdot K_{X}=0$. We claim that $D^{2} \geq 0$. Assume for contradiction that $D^{2}<0$. The twodimensional vector space $\mathrm{NS}(X) \otimes_{\mathbb{Z}} \mathbb{Q}$ is then spanned by $D$ and $K_{X}$, so the Hodge index theorem implies that $K_{X}^{2}>0$, and $X$ must be ruled over $\mathbb{P}^{1}$. In particular $e \geq 0$. Write $D=a C+b f$. Since $D$ is irreducible

$$
D \cdot C=b-a e \geq 0, \quad D \cdot f=a \geq 0,
$$

which is impossible since $D^{2}=a(2 b-e a)<0$.

If $\Phi$ is not a sheaf transform then by Lemma 2.9 we can find a point $y \in Y$ and a cohomology sheaf $E$ of $\Phi\left(\mathcal{O}_{y}\right)$, supported in dimension 1, such that the group $\operatorname{Ext}_{X}^{1}(E, E)$ has dimension at most 1 . Since $E$ is special, any irreducible curve $D$ contained in the support of $E$ satisfies $D \cdot K_{X}=0$, and therefore $D^{2} \geq 0$. But this is a contradiction since the group $\operatorname{Ext}_{X}^{2}(E, E)$ is non-zero, so by Riemann-Roch, $\mathrm{c}_{1}(E)^{2}<0$.

Thus the kernel of $\Phi$ is a sheaf $\mathcal{P}$ on $Y \times X$, flat over $Y$. Given a point $y \in Y$ let $D$ be the support of the sheaf $\mathcal{P}_{y}=\Phi\left(\mathcal{O}_{y}\right)$. If $D$ is zerodimensional, it follows as in Prop. 3.1 that $X$ and $Y$ are isomorphic. Thus we may assume that $D$ is a curve. Then $D \cdot K_{X}=0$ and $D^{2}=0$, and since $\mathcal{P}_{y}$ is simple, $D$ is connected. Furthermore $D$ is irreducible since any irreducible component $D_{0} \subset D$ satisfies $D_{0} \cdot K_{X}=0$ and hence $D_{0}^{2} \geq 0$.

Fix a smooth hyperplane section $H$ on $Y$, with $H \cdot K_{Y} \neq 0$, and let $\Psi: \mathrm{D}(X) \rightarrow \mathrm{D}(Y)$ be a quasi-inverse of $\Phi$. For any point $x \in X$, the support of $\Psi\left(\mathcal{O}_{x}\right)$ meets $H$ at a finite set of points because $\Psi\left(\mathcal{O}_{x}\right)$ is special. We show that for some integer $d$ this defines a map $X \rightarrow$ $\operatorname{Sym}^{d}(H)$ which is an elliptic fibration on $X$.

Recall the definition of the derived dual

$$
\mathcal{O}_{H}^{\vee}=\mathbf{R} \mathcal{H o m}_{\mathcal{O}_{Y}}\left(\mathcal{O}_{H}, \mathcal{O}_{Y}\right)=\mathcal{O}_{H}(H)[-1] .
$$

For any line bundle $L$ on $Y$, one has isomorphisms

$$
\begin{gathered}
\operatorname{Hom}_{\mathrm{D}(Y)}^{i}\left(L, \Psi\left(\mathcal{O}_{x}\right) \stackrel{\mathbf{L}}{\otimes} \mathcal{O}_{H}\right)=\operatorname{Hom}_{\mathrm{D}(Y)}^{i}\left(L \otimes \mathcal{O}_{H}^{\vee}, \Psi\left(\mathcal{O}_{x}\right)\right) \\
=\operatorname{Hom}_{\mathrm{D}(X)}^{i+1}\left(\Phi\left(L \otimes \mathcal{O}_{H}(H)\right), \mathcal{O}_{x}\right) .
\end{gathered}
$$

By the theorem on cohomology and base-change, we can choose $L$ sufficiently ample so that the object

$$
\Phi\left(L \otimes \mathcal{O}_{H}(H)\right)=\mathbf{R} \pi_{X, *}\left(\mathcal{P} \otimes \pi_{Y}^{*}\left(L \otimes \mathcal{O}_{H}(H)\right)\right)
$$

is concentrated in degree 0 and is locally free. Then the above groups vanish unless $i=-1$, so for each $x \in X$, the object

$$
\left.\Psi\left(\mathcal{O}_{x}\right)[-1]\right|_{H}=\Psi\left(\mathcal{O}_{x}\right)[-1] \stackrel{\mathbf{L}}{\otimes} \mathcal{O}_{H}
$$

is concentrated in degree 0 . If the kernel of the transform $\Psi[-1]$ is the object $\mathcal{Q}$ of $\mathrm{D}(Y \times X)$, this implies [8, Lemma 4.3] that $\left.\mathcal{Q}\right|_{H \times X}$ is a 
sheaf on $H \times X$, and is flat over $X$. Thus $\mathcal{Q}$ defines a family of torsion sheaves on $H$, parameterised by $X$, so induces a morphism

$$
f: X \rightarrow \operatorname{Sym}^{d}(H) \text {. }
$$

By the result of Section 2.7, any fibre of $f$ is the intersection over a finite set of points $y \in H$ of the supports of the sheaves $\mathcal{P}_{y}=\Phi\left(\mathcal{O}_{y}\right)$. Each sheaf $\mathcal{P}_{y}$ is supported on an irreducible curve $D_{y}$ satisfying $D_{y}$. $K_{X}=0$, and by Riemann-Roch, given two points $y_{1}, y_{2} \in Y$ one has $D_{y_{1}} \cdot D_{y_{2}}=0$. It follows that any non-singular fibre of $f$ is an elliptic curve. Applying Stein factorisation gives an elliptic fibration $X \rightarrow S$ onto a smooth curve $S$, and hence a contradiction.

The remaining possibility is that $X$ is the unique rational ruled surface with invariant $e=2$. Then, [15, Cor. 2.18], $C \subset X$ is the only irreducible curve satisfying $C \cdot K_{X}=0$, so the argument of Prop. 3.1 shows that $Y$ is birational to $X$. By Lemma 2.3, $X$ and $Y$ have the same Picard number, so $Y$ is also a rational ruled surface, and hence has no elliptic fibration. Applying what we have already proved to $Y$, we conclude that $Y$ also has invariant $e=2$, so $X$ and $Y$ are isomorphic.

\section{Elliptid surfaces}

Fourier-Mukai transforms for elliptic surfaces were introduced in [7]. We start by reviewing the construction given there. Throughout we fix a surface $X$ and a relatively minimal elliptic fibration $\pi: X \rightarrow C$.

Given an object $E$ of $\mathrm{D}(X)$, one defines the fibre degree of $E$

$$
\mathrm{d}(E)=\mathrm{c}_{1}(E) \cdot f
$$

where $f$ denotes the algebraic equivalence class of a fibre of $\pi$. Let $\lambda_{X / C}$ denote the highest common factor of the fibre degrees of objects of $\mathrm{D}(X)$. Equivalently, $\lambda_{X / C}$ is the smallest number $d$ such that there is a holomorphic $d$-section of $\pi$.

Let $a>0$ and $b$ be integers, with $b$ coprime to $a \lambda_{X / C}$. Then, as was shown in [7], there is a smooth, two-dimensional component

$$
Y=\mathrm{J}_{X / C}(a, b)
$$

of the moduli space of pure dimension one stable sheaves on $X$, the general point of which represents a rank $a$, degree $b$ stable vector bundle supported on a smooth fibre of $\pi$.

There is a natural morphism $Y \rightarrow C$, taking a point representing a sheaf supported on the fibre $\pi^{-1}(p)$ of $X$ to the point $p$, and this morphism is a relatively minimal elliptic fibration. Moreover, there is a universal sheaf $\mathcal{P}$ on $Y \times X$, supported on $Y \times_{C} X$, and the corresponding functor $\Phi_{Y \rightarrow X}^{\mathcal{P}}$ is a FM transform. In [7] these ideas are used to prove the following result. 
Theorem 4.1. Take an element

$$
\left(\begin{array}{ll}
c & a \\
d & b
\end{array}\right) \in S L_{2}(\mathbb{Z})
$$

such that $\lambda_{X / C}$ divides $d$ and $a>0$. Let $Y$ be the elliptic surface $\mathrm{J}_{X / C}(a, b)$ over $C$. Then there exist universal sheaves $\mathcal{P}$ on $Y \times X$, flat over both factors, such that the resulting functor $\Phi=\Phi_{Y \rightarrow X}^{\mathcal{P}}$ is an equivalence of categories satisfying

$$
\left(\begin{array}{c}
\mathrm{r}(\Phi E) \\
\mathrm{d}(\Phi E)
\end{array}\right)=\left(\begin{array}{ll}
c & a \\
d & b
\end{array}\right)\left(\begin{array}{c}
\mathrm{r}(E) \\
\mathrm{d}(E)
\end{array}\right)
$$

for all objects $E$ of $\mathrm{D}(Y)$.

When $a=1$ the elliptic surface $\mathrm{J}_{X / C}(a, b)$ is the relative Picard scheme of R. Friedman [13], which we denote more concisely by $\mathrm{J}_{X / C}(b)$.

Lemma 4.2. For any pair of integers $a, b$, with $b$ coprime to $a \lambda_{X / C}$, there is an isomorphism

$$
\mathrm{J}_{X / C}(a, b) \cong \mathrm{J}_{X / C}(b) .
$$

Proof. The essential point is that, as in [1, Theorem 6], the determinant map gives an isomorphism

$$
\operatorname{det}: \mathcal{M}_{X_{p}}(a, b) \longrightarrow \mathcal{M}_{X_{p}}(1, b)=\operatorname{Jac}^{b}\left(X_{p}\right)
$$

on each smooth fibre $X_{p}$ of $\pi: X \rightarrow C$. Glueing these maps gives the isomorphism (2).

In more detail, let $U \subset C$ be an open subset of $C$ over which the morphism $\pi$ is smooth, and let $\mathcal{P}_{U}$ denote the restriction of $\mathcal{P}$ to the open subset

$$
Y_{U} \times_{C} X_{U} \subset Y \times_{C} X .
$$

For each point $y \in Y_{U}$, the restriction of $\mathcal{P}_{U}$ to the fibre $\{y\} \times_{C} X$ is a rank $a$, degree $b$ vector bundle on the smooth elliptic curve $X_{\pi(y)}$. Therefore $\mathcal{P}_{U}$ is locally free, and we can consider the determinant line bundle $\wedge^{a} \mathcal{P}_{U}$. This parameterises degree $b$ line bundles on the fibres of $\pi$, and hence defines an isomorphism

$$
\mathrm{J}_{X / C}(a, b) \times_{C} U \longrightarrow \mathrm{J}_{X / C}(b) \times_{C} U .
$$

Since both spaces in (2) are relatively minimal over $C$, [2, Prop. III.8.4] implies that they are isomorphic.

Lemma 4.3. For any integer $b$ coprime to $\lambda_{X / C}$, the elliptic surface $Y=\mathrm{J}_{X / C}(b)$ has the same Kodaira dimension as $X$.

Proof. The Euler numbers of $X$ and $Y$ are equal by Lemma 2.3. By [12, Prop. I.3.23] we must show that the base orbifolds of $X$ and $Y$ are diffeomorphic, i.e. that for each point $p \in C$, the multiplicities of the fibres of $X$ and $Y$ over the point $p$ are equal.

Let $\Phi: \mathrm{D}(Y) \rightarrow \mathrm{D}(X)$ be a FM transform as in Theorem 4.1, and fix a point $p \in C$. Let $F_{Y}$ be the fibre of $Y$ over $p$. Thus $\mathcal{O}_{F_{Y}}$ has 
Chern character $(0, f, 0)$. It is easy to check using formula (1) that the object $\Phi\left(\mathcal{O}_{F_{Y}}\right)$ of $\mathrm{D}(X)$ has Chern character $-(0, c f, d)$.

Suppose that $F_{Y}$ is a multiple fibre, so that $F_{Y}=m D_{Y}$ for some positive integer $m$, and let $E=\Phi\left(\mathcal{O}_{D_{Y}}\right)$. Then $E$ is an object of $\mathrm{D}(X)$ supported on the fibre $F_{X}$ of $X$ over $p$, and if the first Chern class of $E$ is $-D_{X}$, we must have $m D_{X}=c f$. But $c$ is coprime to $d$, and $m$ divides $\lambda_{X / C}$ which divides $d$, so it follows that $F_{X}$ has multiplicity at least $m$. By symmetry the multiplicities of the fibres of $X$ and $Y$ over $p$ are equal.

We can now prove the following classification result.

Proposition 4.4. Let $X$ be a minimal surface of non-zero Kodaira dimension, with an elliptic fibration $\pi: X \rightarrow C$. If $Y$ is an FM partner of $X$, then $Y$ is isomorphic to the relative Picard scheme $\mathrm{J}_{X / C}(b)$, for some integer $b$ coprime to $\lambda_{X / C}$.

Proof. Let $f$ be the cohomology class of a fibre of $\pi$. The cohomology class $K_{X}$ is a non-zero rational multiple of $f$, [2, Cor. V.12.3], so the support of any special sheaf on $X$ is contained in a finite number of fibres of $\pi$.

Take $x \in X$ lying on a smooth fibre of $\pi$, and take a point $y \in Y$ such that the support of the object $E=\Phi\left(\mathcal{O}_{y}\right)$ contains $x$. Since $\operatorname{Hom}_{\mathrm{D}(X)}(E, E)=\mathbb{C}$, the support of $E$ is connected, hence equal to the (smooth) fibre of $\pi$ containing $x$. Now the Chern class of $E$ must be $(0, a f,-b)$ for some integers $a$ and $b$, and since

$$
\chi\left(E, \Phi\left(\mathcal{O}_{Y}\right)\right)=\chi\left(\mathcal{O}_{y}, \mathcal{O}_{Y}\right)=1,
$$

Riemann-Roch implies that $a \lambda_{X / C}$ is coprime to $b$. Since $E$ is supported on an elliptic curve, all of its cohomology sheaves are non-rigid, so Lemma 2.9 implies that $E$ has only one non-zero cohomology sheaf. Thus a shift of $E$ is a simple sheaf on an elliptic curve, hence stable.

Let $Y^{+}$be the elliptic surface $\mathrm{J}_{X / C}(b)$, with its relatively minimal elliptic fibration $\pi^{+}: Y^{+} \rightarrow C$. There is a transform

$$
\Psi: \mathrm{D}\left(Y^{+}\right) \longrightarrow \mathrm{D}(X)
$$

which takes the structure sheaf of some point of $Y^{+}$to $E$. Applying Prop. 2.5 to the transform $\Psi^{-1} \circ \Phi$ shows that there is a birational equivalence $f: Y \rightarrow Y^{+}$, such that

$$
\left(\Psi^{-1} \circ \Phi\right)\left(\mathcal{O}_{y}\right)=\mathcal{O}_{f(y)}
$$

for all points $y$ in some open subset of $Y$.

If $X$ has Kodaira dimension 1 , then so do $Y$ and $Y^{+}$, so Remark 2.6 shows that $f$ extends to an isomorphism, completing the proof. The only other possibility is that $X$ is a minimal ruled surface over an elliptic base. In that case $Y$ and $Y^{+}$also have Kodaira dimension $-\infty$, and also have Picard number 2 , so are minimal ruled surfaces over 
an elliptic base. By Prop. 3.2 we may assume that $Y$ has an elliptic fibration $\pi: Y \rightarrow C$.

Consider the full subcategory $\mathrm{D}_{s p}(Y)$ of $\mathrm{D}(Y)$ consisting of objects invariant under twisting by $\omega_{Y}$. The support of any object of $\mathrm{D}_{s p}(Y)$ is contained in the union of a finite number of fibres of $\pi$. By uniqueness of Serre functors (see Lemma 2.1), the FM transform $\Psi^{-1} \circ \Phi$ takes objects of $\mathrm{D}_{s p}(Y)$ to objects of $\mathrm{D}_{s p}\left(Y^{+}\right)$. This says that the birational map $f$ takes fibres of $\pi$ to fibres of $\pi^{+}$, so applying [2, Prop. III.8.4] shows that $f$ extends to an isomorphism.

Remark 4.5. If $\sigma$ is a divisor on $X$ such that $\sigma \cdot f=\lambda_{X / C}$, then twisting by $\mathcal{O}_{X}(\sigma)$ gives an isomorphism

$$
\mathrm{J}_{X / C}(b) \cong \mathrm{J}_{X / C}\left(b+\lambda_{X / C}\right) .
$$

Thus an elliptic surface of non-zero Kodaira dimension has only finitely many FM partners.

The argument of Lemma 4.2 shows that the operation of taking duals gives a birational equivalence, hence an isomorphism

$$
\mathrm{J}_{X / C}(b) \cong \mathrm{J}_{X / C}(-b) \text {. }
$$

Finally note that there is an isomorphism

$$
\mathrm{J}_{X / C}(1) \cong X \text {. }
$$

To see this note that the ideal sheaf of the diagonal in $X \times_{C} X$ is flat over both factors, and hence generates a family of rank 1, degree -1 stable sheaves supported on the fibres of $\pi$.

\section{K3 AND ABELIAN SURFACES}

Let $X$ be an abelian or K3 surface. Following Mukai [22], one introduces the extended Hodge lattice of $X$ by using the formula

$$
\left\langle\left(r_{1}, D_{1}, s_{1}\right),\left(r_{2}, D_{2}, s_{2}\right)\right\rangle=D_{1} \cdot D_{2}-r_{1} s_{2}-r_{2} s_{1}
$$

to define a bilinear form on the cohomology ring

$$
\mathrm{H}^{2 *}(X, \mathbb{Z})=\mathrm{H}^{0}(X, \mathbb{Z}) \oplus \mathrm{H}^{2}(X, \mathbb{Z}) \oplus \mathrm{H}^{4}(X, \mathbb{Z}),
$$

and taking the following Hodge decomposition of $\mathrm{H}^{2 *}(X, \mathbb{C})$

$$
\begin{gathered}
\mathrm{H}^{2 *(0,2)}(X, \mathbb{C})=\mathrm{H}^{0,2}(X, \mathbb{C}), \quad \mathrm{H}^{2 *(2,0)}(X, \mathbb{C})=\mathrm{H}^{2,0}(X, \mathbb{C}), \\
\mathrm{H}^{2 *(1,1)}(X, \mathbb{C})=\mathrm{H}^{0}(X, \mathbb{C}) \oplus \mathrm{H}^{1,1}(X, \mathbb{C}) \oplus \mathrm{H}^{4}(X, \mathbb{C}) .
\end{gathered}
$$

Inside $\mathrm{H}^{2}(X, \mathbb{Z})$ one has two sublattices, the Néron-Severi lattice which is

$$
\mathrm{NS}(X)=\mathrm{H}^{2}(X, \mathbb{Z}) \cap \mathrm{H}^{1,1}(X, \mathbb{C}),
$$

and its orthogonal complement $\mathrm{T}(X)$, the transcendental lattice of $X$. The transcendental lattice inherits a Hodge structure from $\mathrm{H}^{2}(X, \mathbb{Z})$.

A Hodge isometry $\phi$ between the transcendental lattices (or extended Hodge lattices) of two K3 (or abelian) surfaces $X$ and $Y$, is an isometry between the relevant lattices which preserves the Hodge decomposition. 
This last condition is equivalent to the statement that $\phi \otimes \mathbb{C}$ takes the cohomology class of the unique (up to scalar multiples) non-vanishing holomorphic 2-form on $Y$ to the corresponding class on $X$.

To each sheaf (or complex of sheaves) $E$ on $X$ one associates a Mukai vector

$$
v(E)=\left(\mathrm{r}(E), \mathrm{c}_{1}(E), \frac{1}{2} \mathrm{c}_{1}(E)^{2}-\mathrm{c}_{2}(E)-\epsilon \mathrm{r}(E)\right) \in \mathrm{H}^{2 *}(X, \mathbb{Z}),
$$

where $\left(\mathrm{r}(E), \mathrm{c}_{1}(E), \mathrm{c}_{2}(E)\right)$ are the Chern classes of $E$, and $\epsilon$ is 0 or 1 depending on whether $X$ is abelian or K3 respectively. Having done this, the Riemann-Roch formula becomes

$$
\chi(E, F)=-\langle v(E), v(F)\rangle,
$$

for any pair of sheaves (or complexes) $E$ and $F$ on $X$.

By Lemma 2.1 any FM partner of $X$ is also an abelian or K3 surface, and Lemma 2.3 shows that an abelian surface could never be a partner of a K3 surface.

The following theorem is due to Mukai 22] and Orlov [24]. We sketch the proof for the reader's convenience, and to fix ideas for the next section where similar techniques are used.

Theorem 5.1. Let $X$ and $Y$ be a pair of $K 3$ (respectively abelian) surfaces. The following statements are equivalent,

(a) there is a FM transform $\Phi: \mathrm{D}(Y) \rightarrow \mathrm{D}(X)$,

(b) there is a Hodge isometry $\phi^{t}: \mathrm{T}(Y) \rightarrow \mathrm{T}(X)$,

(c) there is a Hodge isometry $\phi: \mathrm{H}^{2 *}(Y, \mathbb{Z}) \rightarrow \mathrm{H}^{2 *}(X, \mathbb{Z})$,

(d) $Y$ is isomorphic to a fine, two-dimensional moduli space of stable sheaves on $X$.

Sketch proof.

(a) $\Longrightarrow$ (b). Any FM transform $\Phi: \mathrm{D}(Y) \rightarrow \mathrm{D}(X)$ induces an isomorphism of vector spaces

$$
\phi: \mathrm{H}^{2 *}(Y, \mathbb{C}) \longrightarrow \mathrm{H}^{2 *}(X, \mathbb{C}),
$$

as in Section 2.3. Since the kernel of $\Phi$ is algebraic, this isomorphism preserves the Hodge decomposition. Mukai checks [22, Lemma 4.7, Theorem 4.9], that $\phi$ preserves the inner product and the integral lattices. It follows that $\phi$ takes $\mathrm{T}(Y)$ into $\mathrm{T}(X)$.

(b) $\Longrightarrow(\mathrm{c})$. This is a consequence of a result of Nikulin [22, Prop. 6.1]. The orthogonal complement of $\mathrm{T}(Y)$ in $\mathrm{H}^{2 *}(Y, \mathbb{Z})$ contains the hyperbolic lattice

$$
H=\mathrm{H}^{0}(Y, \mathbb{Z}) \oplus \mathrm{H}^{4}(Y, \mathbb{Z}),
$$

so any isometry of transcendental lattices extends to an isometry of extended Hodge lattices.

$(\mathrm{c}) \Longrightarrow(\mathrm{d})$. Let

$$
\phi: \mathrm{H}^{2 *}(Y, \mathbb{Z}) \longrightarrow \mathrm{H}^{2 *}(X, \mathbb{Z})
$$


be a Hodge isometry, and put $v=\phi(0,0,1)$. Composing with standard automorphisms of $\mathrm{H}^{2 *}(X, \mathbb{Z})$, obtained either by swapping $H^{0}$ and $\mathrm{H}^{4}$, or by twisting by line bundles, we may assume that $v=(r, \ell, s)$, with $r$ positive, $\ell$ ample, and $s$ coprime to $r$.

Since $v$ is algebraic, we can consider $Y^{+}$, the moduli space of stable sheaves on $X$ with Mukai vector $v$, with respect to the polarization $\ell$. The fact that $v$ is primitive implies that this moduli space is fine [22, Theorem A.6], and the fact that $v^{2}=0$ implies that $Y^{+}$is twodimensional. General results of Mukai show that $Y^{+}$is smooth [20] and non-empty [22, Theorem 5.4], [18, Prop. 6.16, Cor. 6.23].

By Cor. 2.8 there is a FM transform

$$
\Psi: \mathrm{D}\left(Y^{+}\right) \longrightarrow \mathrm{D}(X)
$$

such that for any point $y \in Y^{+}, \Psi\left(\mathcal{O}_{y}\right)$ is the corresponding stable sheaf on $X$. The argument given for $(\mathrm{a}) \Longrightarrow(\mathrm{b})$ shows that $\Psi$ gives rise to a Hodge isometry

$$
\psi: \mathrm{H}^{2 *}\left(Y^{+}, \mathbb{Z}\right) \longrightarrow \mathrm{H}^{2 *}(X, \mathbb{Z})
$$

taking $(0,0,1)$ to $v$. The composite $\psi^{-1} \circ \phi$ is a Hodge isometry

$$
\mathrm{H}^{2 *}(Y, \mathbb{Z}) \longrightarrow \mathrm{H}^{2 *}\left(Y^{+}, \mathbb{Z}\right)
$$

fixing $(0,0,1)$, which therefore restricts to give a Hodge isometry

$$
\mathrm{H}^{2}(Y, \mathbb{Z}) \longrightarrow \mathrm{H}^{2}\left(Y^{+}, \mathbb{Z}\right)
$$

In the K3 surface case, the Torelli theorem shows that $Y$ and $Y^{+}$ are isomorphic, and we are done. Otherwise $X$ is an abelian surface, and [26, Theorem 1] shows that $Y$ is isomorphic to either $Y^{+}$or its dual variety. In either case $Y$ is a FM partner of $X$ since dual abelian varieties have equivalent derived categories by the results of [19]. It follows from Cor. 2.10 that there is a universal family of sheaves $\left\{\mathcal{P}_{y}\right.$ : $y \in Y\}$ on $X$, which we may assume are locally free, and which are simple by Lemma 2.2. Then [18, Prop. 6.16] shows that each bundle $\mathcal{P}_{y}$ is actually stable (with respect to any polarization of $X$ ), so $Y$ is indeed a moduli space of stable sheaves on $X$.

$(\mathrm{d}) \Longrightarrow$ (a). Immediate from Cor. 2.8,

Remark 5.2. Given a FM transform $\Phi: \mathrm{D}(Y) \rightarrow \mathrm{D}(X)$ between K3 surfaces, the theorem implies that $Y$ is a moduli space of stable sheaves on $X$. This does not mean that $\Phi$ is given by the formula $\Phi_{Y \rightarrow X}^{\mathcal{P}}$, with $\mathcal{P}$ a universal sheaf on $Y \times X$. As we mentioned in the introduction, finding the set of FM transforms between two K3 surfaces satisfying the conditions of the theorem is a difficult unsolved problem.

Proposition 5.3. Let $X$ be a K3 or abelian surface. Then $X$ has only a finite number of FM partners. 
Proof. Suppose for contradiction that $X$ has infinitely many FM partners $Y$, and choose two such surfaces $Y_{1}$ and $Y_{2}$, not isomorphic, together with a Hodge isometry

$$
\phi: \mathrm{H}^{2 *}\left(Y_{1}, \mathbb{Z}\right) \longrightarrow \mathrm{H}^{2 *}\left(Y_{2}, \mathbb{Z}\right) .
$$

Since $\phi$ preserves the Hodge decomposition, it induces lattice isomorphisms

$$
\mathrm{T}\left(Y_{1}\right) \longrightarrow \mathrm{T}\left(Y_{2}\right), \quad \mathrm{NS}\left(Y_{1}\right) \oplus H \longrightarrow \mathrm{NS}\left(Y_{2}\right) \oplus H
$$

where $H=\mathrm{H}^{0}(Y, \mathbb{Z}) \oplus \mathrm{H}^{4}(Y, \mathbb{Z})$ is the hyperbolic lattice. The second isomorphism shows that the lattices $\mathrm{NS}\left(Y_{1}\right)$ and $\mathrm{NS}\left(Y_{2}\right)$ have the same genus [23, Theorem 1.3.1, Cor. 1.9.4]. There are only finitely many different lattices of each genus [11, Ch. 10, §3.3], so we may choose $Y_{1}$ so that there are infinitely many possible choices for $Y_{2}$ such that $\mathrm{NS}\left(Y_{1}\right)$ and $\mathrm{NS}\left(Y_{2}\right)$ are isometric. For any such choice we can find a Hodge isometry

$$
f: \mathrm{NS}\left(Y_{1}\right) \oplus \mathrm{T}\left(Y_{1}\right) \longrightarrow \mathrm{NS}\left(Y_{2}\right) \oplus \mathrm{T}\left(Y_{2}\right) .
$$

Fix an abstract lattice $W$ isomorphic to $\mathrm{NS}\left(Y_{1}\right) \oplus \mathrm{T}\left(Y_{1}\right)$. Lattices containing $W$ as a sublattice of finite index are all contained in the dual lattice $W^{*}=\operatorname{Hom}_{\mathbb{Z}}(W, \mathbb{Z})$, and thus correspond to subgroups of the finite abelian group $W^{*} / W$, as in [23, §4]. Obviously there are only a finite number of these, so, changing $Y_{1}$ again, we may assume that there are infinitely many possible choices for $Y_{2}$ such that the lattice extensions

$$
\mathrm{NS}\left(Y_{1}\right) \oplus \mathrm{T}\left(Y_{1}\right) \hookrightarrow \mathrm{H}^{2}\left(Y_{1}, \mathbb{Z}\right), \quad \mathrm{NS}\left(Y_{2}\right) \oplus \mathrm{T}\left(Y_{2}\right) \hookrightarrow \mathrm{H}^{2}\left(Y_{2}, \mathbb{Z}\right)
$$

are isomorphic. But for any such choice, the isometry $f$ extends to a Hodge isometry

$$
\mathrm{H}^{2}\left(Y_{1}, \mathbb{Z}\right) \longrightarrow \mathrm{H}^{2}\left(Y_{2}, \mathbb{Z}\right)
$$

If $X$ is a $\mathrm{K} 3$ surface, the Torelli theorem implies that $Y_{1}$ and $Y_{2}$ are isomorphic. In the case when $X$ is an abelian surface we can apply [26. Theorem 1] to conclude that $Y_{1}$ is isomorphic to $Y_{2}$ or its dual. In both cases we obtain a contradiction, since we claimed there were an infinite number of possible choices for $Y_{2}$.

Remark 5.4. Nikulin's results imply that if a K3 surface has Picard number at least 12 then it has no FM partners other than itself [22, Prop. 6.2]. This result is not true in general; Mukai observes [22, p. 394] that there are K3 surfaces with isometric transcendental lattices (hence equivalent derived categories) but distinct Néron-Severi lattices.

\section{ENRIQUES AND BIELLIPTIC SURFACES}

We conclude our classification of FM transforms by considering surfaces with non-trivial canonical bundle of finite order, i.e. Enriques 
and bielliptic surfaces. Collectively we shall call such surfaces quotient surfaces.

All bielliptic surfaces have exactly two elliptic fibrations, and the general Enriques surface is also an elliptic surface in two different ways. Thus it is possible to generate many examples of non-trivial FM transforms for quotient surfaces by considering compositions of the transforms arising from Theorem 4.1. Further examples were described in [10]. Nonetheless, in this section we shall prove that if $X$ is a quotient surface then any FM partner of $X$ is isomorphic to $X$ itself.

Let $X$ be a quotient surface, and let $n$ be the order of $\omega_{X}$. It is easily seen that there is a surface $\widetilde{X}$, with trivial canonical bundle, such that $X$ is the quotient of $\widetilde{X}$ by a free action of the finite cyclic group $G$ of order $n$. We refer to the quotient map $p_{X}: \widetilde{X} \rightarrow X$ as the canonical cover of $X$. Let

$$
\Phi: \mathrm{D}(Y) \rightarrow \mathrm{D}(X)
$$

be a FM transform. By Lemma 2.1, $\omega_{Y}$ also has order $n$, so has a canonical cover $p_{Y}: \widetilde{Y} \rightarrow Y$. In [10] we proved that there is a lift of $\Phi$ to a FM transform

$$
\widetilde{\Phi}: \mathrm{D}(\widetilde{Y}) \longrightarrow \mathrm{D}(\widetilde{X})
$$

making the following two squares of functors commute

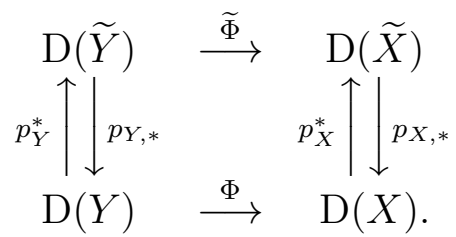

Moreover any such lift is equivariant, in that there is an automorphism $\mu: G \rightarrow G$ such that for each $g \in G$ there is an isomorphism of functors

$$
g^{*} \circ \widetilde{\Phi} \cong \widetilde{\Phi} \circ \mu(g)^{*} .
$$

Proposition 6.1. Let $X$ be an Enriques surface. Then the only FM partner of $X$ is $X$ itself.

Proof. Take notation as above. Thus $\widetilde{X}$ and $\tilde{Y}$ are K3 surfaces and $G=\mathbb{Z} /(2)$. It follows that $Y$ is also an Enriques surface. Let $g$ be the generator of $G$. Define sublattices

$$
\mathrm{H}_{ \pm}^{2 *}(\widetilde{X}, \mathbb{Z})=\left\{\theta \in \mathrm{H}^{2 *}(\widetilde{X}, \mathbb{Z}): g^{*}(\theta)= \pm \theta\right\} .
$$

Note that $\mathrm{H}_{-}^{2 *}(\tilde{X}, \mathbb{Z}) \subset \mathrm{H}^{2}(\tilde{X}, \mathbb{Z})$. Furthermore

$$
\mathrm{H}^{0,2}(\widetilde{X}, \mathbb{C}) \subset \mathrm{H}_{-}^{2 *}(\widetilde{X}, \mathbb{Z}) \otimes \mathbb{C} .
$$

The transform $\widetilde{\Phi}$ induces a $G$-equivariant Hodge isometry between $\mathrm{H}^{2 *}(\widetilde{Y}, \mathbb{Z})$ and $\mathrm{H}^{2 *}(\widetilde{X}, \mathbb{Z})$, and hence gives a $G$-equivariant isometry

$$
f: \mathrm{H}_{-}^{2 *}(\tilde{Y}, \mathbb{Z}) \longrightarrow \mathrm{H}_{-}^{2 *}(\tilde{X}, \mathbb{Z}),
$$


taking the subspace $\mathrm{H}^{0,2}(\widetilde{Y}, \mathbb{C})$ onto $\mathrm{H}^{0,2}(\widetilde{X}, \mathbb{C})$. We claim that $f$ extends to an isometry

$$
f: \mathrm{H}^{2}(\tilde{Y}, \mathbb{Z}) \longrightarrow \mathrm{H}^{2}(\tilde{X}, \mathbb{Z}) .
$$

Assuming this for the moment, note that $f$ is then a $G$-equivariant Hodge isometry, so by the Torelli theorem for Enriques surfaces, [2, VIII.21.2], $X$ and $Y$ are isomorphic.

To prove the claim we use more results of Nikulin. The orthogonal complement of $\mathrm{H}_{-}^{2 *}(\widetilde{X}, \mathbb{Z})$ in $\mathrm{H}^{2}(\widetilde{X}, \mathbb{Z})$, which is equal to $\mathrm{H}_{+}^{2}(\widetilde{X}, \mathbb{Z})$, is even, 2-elementary ([23, Defn. 3.6.1]) and indefinite. The claim then follows from Prop. 1.14.1 and Theorems 3.6.2 and 3.6.3 of [23].

Proposition 6.2. Let $X$ be a bielliptic surface. Then the only FM partner of $X$ is $X$ itself.

Proof. Take notation as above. Then $\widetilde{X}$ is a quotient of a product of two elliptic curves by a finite group $H$ of translations, so is an abelian surface with two elliptic fibrations without multiple fibres. The NéronSeveri group of $\widetilde{X}$ is generated by the algebraic equivalence classes $f_{1}, f_{2}$ of the corresponding fibres 3 and $f_{1} \cdot f_{2}=m$, the order of $H$.

Note that the group $G$ acts on one of the fibres, say $f_{2}$, of $\widetilde{X}$ via multiplication by a complex $n$th root of unity. It follows that $X$ has a multiple fibre of multiplicity $n$, and that there exists a divisor $D$ on $X$ such that $p_{X}^{*} D=f_{1}$.

Consulting the table on [2, p. 148], the possible values of $m$ are 1,2,3 and 4 , and when $m>1$, the prime factors of $m$ are the same as those of $n$. By Remark 4.5, all the relative Picard schemes of $\widetilde{X}$ considered in Section 4 are isomorphic to $\widetilde{X}$. We shall show that $\widetilde{\Phi}$ is isomorphic to a composite of transforms arising from the two elliptic fibrations via Theorem 4.1.

Cor. 2.10 shows that $\widetilde{\Phi}$ is a sheaf transform. Thus we can suppose that for each point $\tilde{y} \in \widetilde{Y}$, the object $\tilde{F}=\widetilde{\Phi}\left(\mathcal{O}_{\tilde{y}}\right)$ is a sheaf on $\widetilde{X}$, of Chern character $\left(r, p f_{1}+q f_{2}, s\right)$ say. The fact that $\widetilde{\Phi}$ is a lift of $\Phi$ implies that

$$
\chi\left(p_{X}^{*}\left(\Phi\left(\mathcal{O}_{Y}\right)\right), \tilde{F}\right)=\chi\left(\Phi\left(\mathcal{O}_{Y}\right), p_{X, *}(\tilde{F})\right)=\chi\left(\Phi\left(\mathcal{O}_{Y}\right), \Phi\left(\mathcal{O}_{y}\right)\right)=1,
$$

where $y=p_{Y}(\tilde{y})$. Now $p_{X}^{*}\left(\Phi\left(\mathcal{O}_{Y}\right)\right)$ has second Chern class divisible by $n$, and hence by $m$, so Riemann-Roch implies that $s$ is coprime to $m$.

Let $h$ be the greatest common divisor of $r$ and $p$, and take integers $b$ and $d$ such that $b p+d r=-h$. Riemann-Roch together with Lemma 2.2 shows that $r s=m p q$. Since $s$ is coprime to $m$, we see that $m$ divides $r / h$. By Theorem 4.1 we can find a transform $\widetilde{\Psi}: \mathrm{D}(\widetilde{X}) \rightarrow \mathrm{D}(\widetilde{X})$ such

\footnotetext{
${ }^{3}$ This statement is false in general, although fortunately Proposition 6.2 is nonetheless correct. See Section 7
} 
that

$$
\left(\begin{array}{c}
\mathrm{r}(\widetilde{\Psi} E) \\
\mathrm{d}(\widetilde{\Psi} E)
\end{array}\right)=\left(\begin{array}{cc}
-p / h & r / m h \\
d m & b
\end{array}\right)\left(\begin{array}{c}
\mathrm{r}(E) \\
\mathrm{d}(E)
\end{array}\right)
$$

where $\mathrm{d}(E)=\mathrm{c}_{1}(E) \cdot f_{2}$ for any object $E$ of $\mathrm{D}(\widetilde{X})$.

The transform $\widetilde{\Psi}$ arises by considering the moduli space of stable sheaves $\widetilde{\Psi}\left(\mathcal{O}_{\tilde{y}}\right)$ on $\widetilde{X}$ of Chern character $\left(0, a f_{2}, b\right)$, where $a=r / m h$. We must show that $\widetilde{\Psi}$ is the lift of a transform $\Psi: \mathrm{D}(X) \rightarrow \mathrm{D}(X)$, it follows from this that $\widetilde{\Psi}$ is $G$-equivariant. By [10, Lemma 5.1], it is enough to check that for some object $A$ of $\mathrm{D}(X)$,

$$
\chi\left(p_{X}^{*} A, \widetilde{\Psi}\left(\mathcal{O}_{\tilde{y}}\right)\right)=1 .
$$

By Riemann-Roch, this is the statement that there is a divisor $D$ on $X$ such that $b$ is coprime to $p_{X}^{*} D \cdot f_{2}$. But we can assume that $p_{X}^{*} D=f_{1}$, and this is enough, since $b$ is coprime to $m$.

Replacing $\Phi$ with the composite transform $\Psi \circ \Phi$ we can now assume that $r=0$ and $p \neq 0$. By Riemann-Roch, $q=0$ also, so $\tilde{F}$ has Chern character $\left(0, p f_{1}, s\right)$, where, as before, $s$ is coprime to $p$ and $m$. There exists an equivariant transform

$$
\widetilde{\Psi}: \mathrm{D}(\tilde{X}) \longrightarrow \mathrm{D}(\widetilde{X})
$$

such that $\Psi\left(\mathcal{O}_{\tilde{x}}\right)$ has this same Chern character, so composing $\widetilde{\Phi}$ with the inverse of $\widetilde{\Psi}$ we can assume that $\tilde{F}=\widetilde{\Phi}\left(\mathcal{O}_{\tilde{y}}\right)$ has Chern character $(0,0,1)$. But $\widetilde{\Phi}$ is a sheaf transform, so there is an isomorphism $\tilde{\phi}: \widetilde{Y} \rightarrow$ $\widetilde{X}$ such that for all $\tilde{y} \in \tilde{Y}$,

$$
\widetilde{\Phi}\left(\mathcal{O}_{\tilde{y}}\right)=\mathcal{O}_{\tilde{\phi}(\tilde{y})} \cdot
$$

Since $\widetilde{\Phi}$ is $G$-equivariant, $\tilde{\phi}$ descends to an isomorphism $\phi: Y \rightarrow X$.

\section{ERratum}

The proof of Proposition 6.2 in the published version of this paper is incorrect, since it assumes that the Néron-Severi group of the canonical cover always has rank 2. We would like to thank Rory Potter and Evgeny Shinder for pointing this out. Fortunately Proposition 6.2 is nonetheless correct. We now explain the proof, much of which is due to Potter Throughout, we denote the group of numerical equivalence classes of divisors on a smooth surface $S$ by $\operatorname{Num}(S)$.

Consider a bielliptic surface $X$ with canonical bundle $\omega_{X}$ of order $n>1$. As in [2, Section V.5], the surface $X$ is a quotient $(A \times B) / J$, with $A$ and $B$ elliptic curves, and $J$ a finite subgroup of the translation group of $A$ which acts faithfully on $B$ by automorphisms. There is a

\footnotetext{
${ }^{4}$ R.D. Potter, Derived autoequivalences of bielliptic surfaces, arxiv 1701.01015; R.D. Potter, Derived categories of surfaces and group actions, PhD thesis, University of Sheffield, 2017, available at http://etheses.whiterose.ac.uk/19643/.
} 
decomposition $J=G \oplus H$, with $G, H \subset J$ both cyclic subgroups, such that $G$ has order $n$, and $H$ acts on $B$ by translations. Writing $k$ for the order of $H$, the possible values of $(n, k)$ are

$$
(2,1), \quad(3,1), \quad(4,1), \quad(6,1), \quad(2,2), \quad(3,3), \quad(4,2) .
$$

The group $H$ acts on $A \times B$ entirely by translations, and the canonical cover $\tilde{X}$ is the quotient $(A \times B) / H$, and is an abelian surface. The surface $\tilde{X}$ has two smooth elliptic fibrations

$$
\tilde{\pi}_{A}: \tilde{X} \rightarrow A / H, \quad \tilde{\pi}_{B}: \tilde{X} \rightarrow B / H .
$$

We denote the classes in $\operatorname{Num}(\tilde{X})$ defined by the fibres of these maps by $\tilde{B}$ and $\tilde{A}$ respectively. Similarly there are elliptic fibrations

$$
\pi_{A}: X \rightarrow A / J, \quad \pi_{B}: X \rightarrow B / J
$$

whose generic fibres define classes $B$ and $A$ in $\operatorname{Num}(X)$ respectively.

Since $H^{2}\left(X, \mathcal{O}_{X}\right)=0$, the Néron-Severi group $\mathrm{NS}(X)$ coincides with $H^{2}(X, \mathbb{Z})$, and the numerical equivalence group $\operatorname{Num}(X)$ is the quotient of $\operatorname{NS}(X)$ by the torsion subgroup. Serrano proved 5 that there are classes $A^{\prime}, B^{\prime} \in \operatorname{Num}(X)$ with $n A^{\prime}=A$ and $k B^{\prime}=B$ such that

$$
\operatorname{Num}(X)=\mathbb{Z} A^{\prime} \oplus \mathbb{Z} B^{\prime} .
$$

Since $A \cdot B=k n$, it follows that $A^{\prime} \cdot B^{\prime}=1$.

The canonical cover $p=p_{X}: \tilde{X} \rightarrow X$ induces a map

$$
p_{*}: \operatorname{Num}(\tilde{X}) \rightarrow \operatorname{Num}(X) .
$$

Consider the image $\Delta=\operatorname{im}\left(p_{*}\right) \subset \operatorname{Num}(X)$ of this map. We claim that given $d \in \mathbb{Z}$ there are implications

$$
d A^{\prime} \in \Delta \Longleftrightarrow n\left|d, \quad d B^{\prime} \in \Delta \Longleftrightarrow k\right| d .
$$

The proofs of the two statements are the same, so we focus on the first. In one direction, it is easy to check that

$$
n A^{\prime}=A=p_{*}(\tilde{A}) \in \Delta .
$$

For the converse, suppose that $d A^{\prime}=p_{*}(C)$ for some class $C \in \operatorname{Num}(\tilde{X})$. Then, since $p^{*}(A)=n \tilde{A}$, and $A \cdot A=0$, it follows that $\tilde{A} \cdot C=0$. A result of Kani ${ }^{6}$ then shows that $C$ is a multiple of $\tilde{A}$, which proves the claim.

Suppose now that we have a FM transform $\Phi: D(Y) \rightarrow D(X)$. We must show that $Y \cong X$. By the existence of the lift of $\Phi$ to the canonical covers, and Cor. 2.10, we know that $\Phi$ is a sheaf transform. Composing

\footnotetext{
${ }^{5}$ Theorem 1.4 in F. Serrano, Divisors of bielliptic surfaces and embeddings in $\mathbb{P}^{4}$, Math. Z., 203, 527 - 533, (1990).

${ }^{6}$ Proof of Proposition 2.3 in E. Kani, Elliptic curves on Abelian surfaces, Manuscripta Math. 84, 199 - 223 (1994).
} 
with a shift we can therefore suppose that $\Phi$ takes skyscrapers of points on $Y$ to sheaves on $X$ of class

$$
v=\left[\Phi\left(\mathcal{O}_{y}\right)\right]=\left(r, a A^{\prime}+b B^{\prime}, s\right) \in \mathbb{Z} \oplus \operatorname{Num}(X) \oplus \mathbb{Z} .
$$

We have $r \geq 0$. The condition $\chi(v, v)=0$ gives $r s=a b$. By the existence of the lift, $\Phi\left(\mathcal{O}_{y}\right)$ is the push-forward of an sheaf on $\tilde{X}$. It follows that $n \mid r$ and $a A^{\prime}+b B^{\prime} \in \Delta$.

Suppose first that $r=0$. Then either $a=0$ or $b=0$. It follows that $\Phi\left(\mathcal{O}_{y}\right)$ is supported on a fibre of one of the two elliptic fibrations $\pi_{A}, \pi_{B}$. Let us denote this fibration by $\pi: X \rightarrow C$. The argument of Prop. 4.4 then applies, and shows that $Y=J_{X / C}(b)$, with $b$ coprime to $\lambda=\lambda_{X / C}$. Since $A^{\prime} \cdot B=k$ and $A \cdot B^{\prime}=n$, we must have

$$
\lambda \in\{1,2,3,4,6\} .
$$

But the only units in $\mathbb{Z} / \lambda \mathbb{Z}$ are then \pm 1 , so it follows from the arguments of Remark 4.5 that $Y \cong X$.

Suppose then that $r>0$. Consider the elliptic fibration

$$
\pi_{A}: X \rightarrow A / J
$$

whose fibres have class $B$. The divisor $A^{\prime}$ gives a $k$-section. Sheaves of class $v$ have rank $r$ and fibre degree $k a$. Take $x, y \in \mathbb{Z}$ with

$$
x r+y k a=h:=\operatorname{gcd}(r, k a) .
$$

We claim that we can suppose that $k \mid x$. Assuming this, consider

$$
\left(\begin{array}{cc}
k a / h & -r / h \\
x & y
\end{array}\right)\left(\begin{array}{c}
r \\
k a
\end{array}\right)=\left(\begin{array}{l}
0 \\
h
\end{array}\right)
$$

The negative of the matrix on the left is then as in Theorem 4.1, so lifts to a relative Fourier-Mukai transform. Since all relative Jacobians of $X$ are isomorphic to $X$ as above, we obtain an equivalence

$$
\Psi: D(X) \rightarrow D(X)
$$

which takes $v$ to a vector of rank 0 . Applying the argument above to $\Psi \circ \Phi$ we conclude that $Y \cong X$, which completes the proof.

To prove the claim we can assume that $k \in\{2,3\}$ is prime. Writing $r=h r^{\prime}$ and $k a=h t^{\prime}$ we are looking for pairs $x, y \in \mathbb{Z}$ with $x r^{\prime}+y t^{\prime}=1$. If $(x, y)$ is such a pair, so is $\left(x+t^{\prime}, y-r^{\prime}\right)$. Thus we can find such a pair with $k \mid x$ precisely if $k \nmid t^{\prime}$. So what we must prove is that the highest power of $k$ which divides $k a$ also divides $r$.

Consulting the list of possibilities, we either have $n=k \in\{2,3\}$ or $(n, k)=(4,2)$. Recall that

$$
a A^{\prime}+b B^{\prime} \in \Delta, \quad r s=a b, \quad n \mid r .
$$

Clearly, one of $a$ or $b$ is divisible by $k$. In the cases when $n=k$, using (3), it follows that since one of the two classes $a A^{\prime}, b B^{\prime}$ lie in $\Delta$, they both do, and hence $k$ divides both $a$ and $b$. In the remaining case $(n, k)=(4,2)$ we have $4 \mid a b$, and we again conclude that $k$ divides both 
$a$ and $b$. The primitivity of $v$ now ensures that $k \nmid s$, and the claim follows.

\section{REFERENCES}

[1] M. Atiyah, Vector bundles over an elliptic curve, Proc. London Math. Soc. 7 (1957), 414-452.

[2] W. Barth, C. Peters, A. Van de Ven, Compact Complex Surfaces, Ergebnisse Math. Grenzgeb. (3), vol. 4, Springer-Verlag, 1984.

[3] C. Bartocci, U. Bruzzo, D. Hernández Ruipérez, A Fourier-Mukai transform for stable bundles on K3 surfaces, J. reine Angew. Math. 486 (1997) 1-16.

[4] A.I. Bondal, M.M. Kapranov, Representable functors, Serre functors, and mutations, Math. USSR Izvestiya 353 (1990) 519-541.

[5] A.I. Bondal, D.O. Orlov, Semiorthogonal decomposition for algebraic varieties, Preprint (1995) alg-geom 9506012.

[6] A.I. Bondal, D.O. Orlov, Reconstruction of a variety from the derived category and groups of autoequivalences, Preprint (1997) alg-geom 9712029.

[7] T. Bridgeland, Fourier-Mukai transforms for elliptic surfaces, J. reine angew. math. 498 (1998) 115-133, alg-geom 9705002.

[8] T. Bridgeland, Equivalences of triangulated categories and FourierMukai transforms, Bull. London Math. Soc. 31 (1999), no. 1, 25-34, alg-geom 9809114.

[9] T. Bridgeland, Fourier-Mukai transforms for surfaces and moduli spaces of stable sheaves, Ph.D. thesis, Edinburgh University (1998).

[10] T. Bridgeland, A. Maciocia, Fourier-Mukai transforms for quotient varieties, Preprint (1998) alg-geom 9811101.

[11] J. Cassels, A. Frölich, editiors, Algebraic number theory (Proc. Instructional Conf., Brighton), Academic Press, London (1967).

[12] R. Friedman, J. Morgan, Smooth four-manifolds and complex surfaces, Ergebnisse Math. Grenzgeb. 3 27, Springer-Verlag, Berlin (1994).

[13] R. Friedman, Vector bundles and $S O(3)$-invariants for elliptic surfaces, Journal Amer. Math. Soc. 81 (1995) 29-141.

[14] R. Hartshorne, Residues and duality, Lect. Notes Math. 20, Springer-Verlag, 1966.

[15] R. Hartshorne, Algebraic Geometry, Grad. Text Math. 52, Springer-Verlag, 1977.

[16] M. Kontsevich, Homological algebra of mirror symmetry, Proc. of the I.C.M., Vol. 1, 2 (Zürich, 1994), 120-139, Birkhuser, Basel, 1995, alg-geom 9411018.

[17] A. Maciocia, Generalized Fourier-Mukai transforms, J. reine angew. Math. 480 (1996) 197-211, corrected version alg-geom 9705001.

[18] S. Mukai, Semi-homogeneous vector bundles on an abelian variety, J. Math Kyoto Univ. 182 (1978) 239-272.

[19] S. Mukai, Duality between $D(X)$ and $D(\hat{X})$ with its application to Picard sheaves, Nagoya Math. J. 81 (1981), 153-175.

[20] S. Mukai, Symplectic structure of the moduli space of sheaves on an abelian or K3 surface, Invent. math. 77 (1984), 101-116.

[21] S. Mukai, Fourier functor and its application to the moduli of bundles on an abelian variety, Adv. Pure Math. 10 (1987), 515-550.

[22] S. Mukai, On the moduli space of bundles on K3 surfaces I, in: Vector Bundles on Algebraic Varieties, M.F. Atiyah et al., Oxford University Press (1987), 341413.

[23] V.V. Nikulin, Integral symmetric bilinear forms and some of their applications, Math. USSR Izvestiya, 141 (1980) 103-167. 
[24] D.O. Orlov, Equivalences of derived categories and K3 surfaces, J. Math. Sci. (New York) 845 (1997) 1361-1381, alg-geom 9606006.

[25] D.O. Orlov, On equivalences of derived categories of coherent sheaves on abelian varieties, Preprint (1997) alg-geom 9712017.

[26] T. Shioda, The period map of abelian surfaces, J. Faculty Sci. Univ. Tokyo, Sect. 1A 251 (1979) 47-59. 